\title{
Between Science, Technology and Society
}

\author{
Alessandro Deserti and Francesca Rizzo
}

The intersection and permeability of science, innovation and society result in a series of benefits and challenges, underlying the important role the latter can and should play. The following paragraphs present the theoretical background and the objectives of the SISCODE (Society in Innovation and Science through CO-DEsign) project investigating this interconnection, the issues that emerged through its journey and the results gained. Therefore, it frames the knowledge obtained throughout the threeyear duration of the project, situating the notion of Responsible Research and Innovation (RRI) in the co-creation domain, and introducing the issues that emerge when moving from the theoretical concept to practice [1, 2]. It inspects how co-creation and design knowledge and tools can be applied to engage citizens in shaping solutions that are meant to be more inclusive, responsible and sustainable, and how these approaches and methodologies could be applied to operationalize RRI. Particular attention is drawn to how small-scale experimentations can lead to significant scalein, scale-up and scale-out processes. The book will show how these processes can lead to organizational learning and transformation, but also how they can provide evidence-based knowledge which nurtures policy making processes with the potential of achieving broader societal impacts in Science, Technology and Innovation (STI) policy making [3]. Investigating the benefits and implications of applying participatory research and innovation approaches in society, this chapter embraces a context-sensitive perspective [4] and explores the crossroads of diverse forms of innovation: not only research-driven but also practice-based, and not only technological but also social. This reasoning provided the theoretical background which led to the construction of a learning framework, adopted as a guide for the 10 co-creation labs in which the real-life experimentations described in this volume were conducted.

\footnotetext{
A. Deserti $(\varangle) \cdot$ F. Rizzo

Department of Design, Politecnico di Milano, 20158 Milan, Italy

e-mail: alessandro.deserti@polimi.it
} 


\section{Areas of Interest of the SISCODE Project}

SISCODE combined diverse fields of study and areas of work. In particular, the research and innovation project investigated the relationship between RRI and cocreation, with a specific focus on STI policy making. These distinct matters have been reconnected in theory and practice, identifying a potentiality of achieving positive results and impacts when applying co-creation approaches, methodologies and tools to operationalize RRI [5].

\section{Responsible Research and Innovation}

Innovation and science are powerful drivers when it comes to the development of all factors that influence modern society and therefore the direction of transformation of societies and all the single individuals that are a part of it [6]. The recognition of this influence has led to the emergence of a new approach in the fields of science, research and innovation, to make them more responsible impacting STI policy making. The emergence of the approach within the framework and context of European policy making dates back to 2011 having been introduced as a topdown approach for research policy which contrasts with the concept itself promoting bottom-up initiatives and pathways to innovation [7].

RRI entails the transition from solutions developed internally within the research community and only tolerated passively by society towards ones that are taking citizens and other actors actively into consideration as part of the development of solutions that are more apt to achieve desirable results with a high impact [6].

This reflection on the societal impact of innovation calls for a change in innovation processes and a shift of roles of its actors, including all players into the innovation process, which should lead to sharing and redefining power, privileges and responsibilities [2]. Apart from the aspect of inclusion, RRI aims to anticipate impacts by analyzing the contexts of implementation and taking into account all the actors and factors that influence the implementation of a solution. Furthermore, findings throughout the development are planned to impact on the process itself, making it more reflective, flexible and responsive to new insights and perspectives [8].

Witnessing this shift towards the involvement of citizens and other actors in the innovation process, it is necessary to understand its potentialities as well as its implications: this calls for new approaches, techniques, processes and mindsets for the effective integration and involvement of society in innovation.

Despite having been widely discussed in theory as a relevant opportunity to move towards more sustainable futures [9], there's still a lack of evidence of impacts of RRI in empirical settings, which leaves open issues especially in terms of contextsensitivity and translation from theory into practice for real and measurable impact [10].

It has been recognized that the full adoption of RRI requires an in-depth transformation in organizations and ecosystems or institutional settings, to be embedded as a general approach towards innovation that requires the reflection not only on the outcomes of innovation itself but also the purpose and process of innovating leading to a shift in the overall mindset and way of working. 
The scientific and technological advancement and the responsibility related to it discussed in RRI directly refers to the substantial societal challenges that are being tackled with innovation [2].

\section{Co-creation}

Co-creation has received significant attention in the context of innovation in recent years, in particular as a part of the field of participatory design. It has been identified as a potential booster for the implementation of new and experimental solutions due to both its practicality and its versatility in adapting to diverse and changing environments and contexts [11].

One of the central points of co-creation is the transformation of passive actors like end-users into operating ones, involving them actively in the development processes of products, services and systems [12] to define and create value commonly and taking all actors and their needs into account [13].

Co-creation considers users and actors not only during research phases, but aims to actively involve them across the phases of ideation in co-design processes until the prototyping and implementation of a solution, thus including co-production [14].

From a business point of view, this active involvement in participatory processes usually aims at the co-creation of value, shifting the focus from a business-centric one towards personalised and satisfying customer experiences [15].

These characteristics led to expanding the fields of application as well as the notion of co-creation. In particular, it has been experimented as a promising means to engage neglected actors and stakeholders in other fields of innovation (e.g. in public sector innovation) and as a way to set up collaborative processes like those that are needed to better include society in innovation [5].

The SISCODE project explored this pathway of operationalizing RRI through co-creation to investigate the potentialities, opportunities and barriers of co-creation in the RRI context. In particular, the project analysed the favorable conditions for co-creation, the dynamics activated during the process of adoption of co-creation, and how capacities for co-creation in organisations are built.

\section{The SISCODE Project and Its Objectives}

SISCODE (Society in Innovation and Science through CO-DEsign) is a threeyear EU-funded project within the Horizon 2020 programme with 17 cross-sector partners, completed in April 2021.

It aimed to explore the application of co-creation, and co-design specifically, for the operationalization of RRI in different contexts.

Its investigation is based on the triangulation of the results of different but interconnected research streams: the theoretical framing of the single areas of work (primarily RRI, co-creation and policy making) and their interconnection; the analysis of existing cases where co-creation has been applied in the context of RRI in Europe and beyond; and finally, the conduction of ten real-life experimentations. For the conduction of the experimentation, an analytical, reflective learning framework 
was developed to explore the provoked shifts and transformations in projects and organizations, as well as in policies and policy making processes triggered by the interaction between citizens, stakeholders and policy makers. Therefore, the project frames the knowledge obtained throughout the three years of the project, situating the notion of RRI in the co-creation domain, and introducing issues that emerge when moving from the theoretical concept to practice [1,2].

\section{Objectives}

To grasp and further explore the circulation and establishing of the phenomenon of co-creation as an approach for bottom-up and design-driven development as well as its potential for replication and scaling when applied in the context of RRI, the SISCODE project was carried out according to three main objectives:

1. The production of a study extended across Europe to investigate existing cocreation ecosystems at different scales ranging from local and regional to national levels and identify and extract patterns of dynamics, drivers and barriers encountered when integrating society in science and innovation. It specifically addressed the cultural, organisational, institutional and regulatory conditions that may favour or hinder co-creation. Furthermore, particular attention was posed to the engagement of stakeholders, the techniques and dynamics of their involvement and how their diversity influenced and affected the process and the final solution.

2. The experimentation of (co-)design not only as an approach, but also as a set of skills and competences, to see how the building of these capacities can be favoured and supported to enable the application co-creation in RRI and STI policy making.

3. The understanding of the transformation needed beyond the development of capacities in terms of organisational, procedural and cultural shifts for the permanent and stable embedding of co-creation in organisational processes and culture and how eventual barriers identified can be overcome.

In essence, SISCODE aimed to explore the operationalization of RRI by investigating the application of co-creation to reach this goal, starting from the theoretical background and existing cases to then conduct its own transnational experimentation across Europe.

This book describes this system of co-creation labs and provides insights drawn from their experimentation of applying co-creation in their single contexts while being in constant exchange with each other, with the networks that they created to conduct the experimentation and with the other partners in the research consortium, to foster peer-to-peer learning and cross-fertilisation. 


\section{RRI in SISCODE-From Theory to Practice through Co-creation}

SISCODE investigated how knowledge, methodologies and tools from the field of design can be applied to shape concrete solutions to relevant societal challenges towards Responsible Innovation taking the inclusivity, responsibility and sustainability of these solutions into account.

The activities conducted are aimed to function as a bridge for the identified gap between theory and practice in RRI through the collaborative development of specific solutions.

In these processes, citizens and other stakeholders are engaged to collaboratively develop solutions for specific local and global problems. The research project investigated and reflected upon the broader transformations triggered by the experimentations and the exchange within the project, both at an organisational level of the single labs as well as within their surrounding ecosystem.

Co-creation has been applied as a means to deal with and overcome the barriers identified in the operationalization of RRI and to trigger the shift within organisations needed to fully embed the new approach to then influence the entire ecosystem.

A series of activities were planned and conducted to support these processes in the frame of the project and provide concrete support to the pilots:

\section{- Training}

Knowledge on co-creation was transmitted in specific training sessions, providing background knowledge, tools for the conduction of co-creation activities, like canvases, cards and instruction, and building capacities for the planning, conduction and facilitation of workshops and other co-creation activities.

\section{- Opportunities for peer-to-peer learning}

Acknowledging the diversity of the pilots and the influence of these differences and the entirely distinct contexts, confrontation has been identified as an opportunity to exchange best practices, ideas and collaboratively find solutions to specific problems. For this reason, regular meetings and calls have been organised as a space for interrelation, conversation and peer-to-peer learning.

\section{- Dialogue between researchers and practitioners}

Recognizing the gap between theory and practice not only identified in literature but in the project itself among academic partners and practitioners, a series of meetings have been organised to discuss specific research topics from the various points of view, aiming to bridge this gap within the project and identifying points of connection and dialogue between researchers and practitioners.

\section{- Reporting as an instrument for self-reflection}

Material to be produced for reporting and assessing the experimentation has been mainly collected following templates composed by a series of reflective questions to trigger reflections on the conducted activities and ongoing transformations while reporting them.

A learning framework, described in detail in Chap. 2, was set up to support and guide this process of moving from theory to practice having all pilots following the 
same general framework adapting its elements to the specific context and conditions. This is relevant in terms of reacting to the previously identified importance of the context while preserving the possibility to still assess and compare the single experimentations notwithstanding their diversity.

The overall project adopted an approach to place these small-scale experiments within larger ecosystems of co-creation exploring opportunities for scaling and reconnect the findings to the general issues identified during the initial desk research.

\section{The Importance of Small-Scale Experiments}

The necessity of impacting ecosystems on a broader scale to influence policies requires impact at not only local, but regional, national and international levels [16]. Small-scale pilots have been identified as a potential to experiment new approaches and concepts to then 'scale, what works' [17].

The advantage of pilots conducted on a smaller scale is not only related to their feasibility but also to their focus on a limited and very specific environment adopting a sensitive perspective in relation to the surrounding context [4]. This contextsensitivity becomes particularly relevant when investigating RRI initiatives where significant levels of context-dependence have been found as one of the barriers for implementation [13, 18-20].

This aspect underlines both the importance of small-scale experiments conducted in very specific contexts to then make considerations on their scaling as well as the necessity to consider these scaling processes and integrate them into pilots like the ones conducted in SISCODE from the very beginning.

Moore et al. have divided the scaling process into three different elements, scaling up, scaling out and scaling deep, and all three of them combined are necessary to impact larger systems [16].

- Scaling out refers to the wider dissemination and replication of the solution to impact a larger number of addressants in this way [16]. In SISCODE, this dimension has been addressed with a variety of dissemination activities in each lab together with business model workshops and considerations on replication to reflect and collect feedback on opportunities of scaling the single solutions out beyond the project context.

- Scaling up relates directly to the influence on laws and policies transforming existing institutions [16]. The pilots have addressed this dimension seeking direct contact, exchange and confrontation specifically with policy makers and decision makers in their respective field of work to collaboratively understand barriers and opportunities within the current policy framework together with potentialities to influence and transform this framework participating and contributing in the shaping of new policies. 
Here it is worth to be mentioned, that especially the value of evidence-based knowledge has been explored to reach out to decision makers to achieve broader impacts on society.

- Scaling deep introduces culture and mindset as an additional dimension to be influenced to achieve impact at a greater scale. The cultural and visionary shift that is required to deeply embed a new solution, its mindset and approach to ensure not only its integration in a context but also create a fertile ground for replication and scaling with the involved actors eventually becoming advocates to further distribute innovation.

Particular attention has been posed at this dimension in SISCODE investigating the changes in mindset and way of working, that the pilot has triggered both in the organisation and the surrounding ecosystem together with the dynamics of these transformations.

\section{Levels and Dimensions of Investigation}

The specific levels investigated in SISCODE range from the micro and meso up to the macro level. While the micro level refers to the internal activities and dynamics as well as the immediate surroundings of an organisation, the meso level zooms out to networks of stakeholders and bigger groups often still limited to a regional level, while the macro level takes a focus on national and institutional governance processes up to transnational dynamics and systems [21].

While the experimentations did mainly take place and directly impacted on a micro-level, the project explored and reflected on how each of the experimental solutions could be scaled or replicated to influence systems on meso- and even macro levels.

These levels of analysis are taken up in the final chapter, the comparative analysis, where the ten experimentations conducted are compared identifying essential differences and common aspects with a specific focus on policies and policy making when applying co-creation in RRI contexts, reconnecting them to the theoretical background of the project by drawing initial conclusions on barriers and opportunities considering a wider scale from a future perspective.

The following chapter presents the empirical reasoning at the ground of the experimentation and its methodology with the learning framework set up to plan, conduct and monitor the pilots. In particular, it shows how the process has been established to support the tackling of challenges for the single organisations in terms of stakeholder engagement, dealing with communities and society and managing transformations.

\section{References}

1. Von Schomberg L, Blok V (2018) The turbulent age of innovation. Synthese, pp 1-17 
2. Von Schomberg R (2013) A vision of responsible research and innovation. In: Owen R, Bessant J, Heintz M (eds) Responsible innovation. Wiley, Chichester, pp 51-74

3. Deserti A, Rizzo F, Smallman M (2020) Experimenting with co-design in STI policy making. Policy Des Pract 3(2):135-149

4. Bekkers V, Tummers LG, Stuijfzand BG, Voorberg W (2013) Social innovation in the public sector: an integrative framework. LIPSE Working articles, 1

5. Bajmócy Z, Pataki G (2019) Responsible research and innovation and the challenge of cocreation. In: Responsible research and innovation and the challenge of co-creation (in press)

6. Owen R, Bessant J, Heintz M (eds) (2013) Responsible innovation: managing the responsible emergence of science and innovation in society. Wiley, Chichester

7. Zwart H, Landeweerd L, van Rooij A (2014) Adapt or perish? Assessing the recent shift in the European research funding arena from 'ELSA' to 'RRI.' Life Sci, Soc Policy 10(1):1-19

8. Stilgoe J, Owen R, Macnaghten P (2013) Developing a framework for responsible innovation. Res Policy 42:1568-1580

9. European Commission: Responsible research and innovation. https://ec.europa.eu/pro grammes/horizon2020/en/h2020-section/responsible-research-innovation. Last accessed 2021/03/28

10. European Commission (2015) Directorate-general for research and innovation: indicators for promoting and monitoring responsible research and innovation: report from the expert group on policy indicators for responsible research and innovation. Publications Office, Luxembourg

11. Payne AF, Storbacka K, Frow P (2008) Managing the co-creation of value. J Acad Mark Sci 36(1):83-96

12. Saarijärvi H (2012) The mechanisms of value co-creation. J Strateg Mark 20:381-391

13. Rizzo F, Deserti A, Komatsu TT (2020) Implementing social innovation in real contexts. Int J Knowl Based Dev 11(1):45-67

14. Frow P, Nenonen S, Payne A, Storbacka K (2015) Managing co-creation design: a strategic approach to innovation: managing co-creation design. Br J Manag 26(3):463-483

15. Prahalad CK, Ramaswamy V (2004) Co-creation experiences: the next practice in value creation. J Interact Mark 18:5-14

16. Moore M-L, Riddell D, Vocisano D (2015) Scaling out, scaling up, scaling deep: strategies of non-profits in advancing systemic social innovation. The J Corp Citizensh 58:67-84

17. Bradach J, Grindle A (2014) Emerging pathways to transformative scale. In: Smarter philanthropy for greater impact: rethinking how grantmakers support scale. Supplement to 'Stanford Social Innovation Review.'

18. Deserti A, Rizzo F (2019) Embedding design in the organizational culture: challenges and perspectives. In: Design culture: objects and approaches, pp 39-51

19. Deserti A, Rizzo F (2019) Context dependency of social innovation: in search of new sustainability models. Eur Plan Stud 28(5):864-880

20. Howlett M (2014) From the 'old' to the 'new' policy design: design thinking beyond markets and collaborative governance. Policy Sci 47(3):187-207

21. Rizzo F, Deserti A, Crabu S, Smallman M, Hjort J, Hansen SJ, Menichinelli M (2018) Co-creation in RRI practices and STI policies. SISCODE deliverable D1.2. https://ec.eur opa.eu/research/participants/documents/downloadPublic?documentIds $=080166 \mathrm{e} 5 \mathrm{bedc} 3 \mathrm{a} 0 \mathrm{~d} \&$ appId=PPGMS. Last accessed 2021/03/21. 
Open Access This chapter is licensed under the terms of the Creative Commons Attribution 4.0 International License (http://creativecommons.org/licenses/by/4.0/), which permits use, sharing, adaptation, distribution and reproduction in any medium or format, as long as you give appropriate credit to the original author(s) and the source, provide a link to the Creative Commons license and indicate if changes were made.

The images or other third party material in this chapter are included in the chapter's Creative Commons license, unless indicated otherwise in a credit line to the material. If material is not included in the chapter's Creative Commons license and your intended use is not permitted by statutory regulation or exceeds the permitted use, you will need to obtain permission directly from the copyright holder.

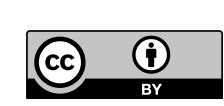

